\title{
Deterioration of tensile behavior of concrete exposed to artificial acid rain environment
}

\author{
Y.F. Fan*1, Z.Q. $\mathrm{Hu}^{2}$ and H.Y. Luan ${ }^{1}$ \\ ${ }^{1}$ Institute of Road and Bridge Engineering, Dalian Maritime University, Dalian, China \\ ${ }^{2}$ Institute of Civil and Hydraulic Engineering, Dalian University of Technology, Dalian, China
}

(Received May 3, 2011, Revised July 16, 2011, Accepted February 16, 2012)

\begin{abstract}
This study is focused on evaluation of the tensile properties of concrete exposed to acid rain environment. Acid rain environment was simulated by the mixture of sulfate and nitric acid in the laboratory. The dumbell-shaped concrete specimens were submerged in pure water and acid solution for accelerated conditioning. Weighing, tensile test, CT, SEM/EDS test and microanalysis were performed on the specimens. Tensile characteristics of the damaged concrete are obtained quantitatively. Evolution characteristics of the voids, micro cracks, chemical compounds, elemental distribution and contents in the concrete are examined. The deterioration mechanisms of concrete exposed to acid rain are well elucidated.
\end{abstract}

Keywords: concrete; multiscale; SEM; atmospheric corrosion.

\section{Introduction}

Pollution of acid rain was firstly observed in 1852. Presently, acid rain has become to be an increasing global environmental issue. Northeast America, Central Europe and China have been mentioned as the three largest acid rain-prone regions in the world (Zhang 2011, Menz 2004, Ito 2000, Wang 2002). As a consequence, large amounts of concrete structures are subjected to the aggressive environment which was neither anticipated nor taken into consideration at the construction stage (Kong 1987). At present, the negative effect of acid rain environment on the durability of infrastructural facilities and the historic buildings has been the focus of worldwide attention.

It is evident that the deterioration of construction materials will lead to a fast degradation of the structures. Therefore, it is essential to have a good understanding of deterioration mechanism of concrete in severe environment. The deteriorating process of concrete is determined by the mutual interaction of many factors, such as the complex servicing condition, chemical composition and its mechanical behavior, permeability, microstructure of the construction materials etc. (Zivica 2002). In the previous surveys, the used methods and tests include damage to the appearance, mechanical properties, chemical composition, pore structure and microstructure composition of concrete specimen (Zivica 2004). To obtain the characteristic change of the mechanical property (i.e., weight,

\footnotetext{
* Corresponding author, Professor, E-mail: fanyf72@yahoo.com.cn
} 
compressive strength, flexural strength, elastic modulus etc.) with the time of the action of acidic medium (such as $\mathrm{HCl}, \mathrm{SO}_{2}$ and $\mathrm{NO}_{\mathrm{x}}$ etc.), experimental studies were performed (Kong 1987, Fattuhi 1998, Pavlik 1994, Sersale 1998, Xie 2004, Huang 2005, Fan 2010). Considering that concrete has a highly heterogeneous microstructure and exceedingly complex composite behavior, multi-scale analysis becomes necessary to obtain a profound understanding of deformation and failure mechanism. To well describe the microstructural changes occurring in concretes exposed to a variety of severe environments, a broad range of analytical techniques (such as SEM, X-ray diffraction technology, EDX etc.) are used successfully to identify the cause of deterioration (Zivica 2002, Grattan-Bellew 1996, Rendell 1999, Cnudde 2009, Girardi 2010). Unfortunately, the reported research has been carried out using separate single scale analysis. Although tensile property is a crucial mechanical characteristic for concrete, and will have a significant effect on the cracking, durability of the concrete structures in practical engineering, until recently few references deal with the deteriorating process of the tensile behavior of concrete induced by the various severe environments.

In this paper, tensile properties of concrete exposed to acid rain environment were studied from its multi-scale behaviors. Batches of dumbbell-shaped concrete specimen were cast for the tensile test. Artificial acid rain environment with $\mathrm{pH} 1.0$ level were made by the mixture of sulfate and nitric acid solutions in the laboratory. After being exposed to the simulated acid rain solutions for various periods, tensile test, computerized tomography (CT), scanning electron microscope (SEM) and energy dispersive spectroscopy (EDS) analysis were performed on the concrete specimens at various damaged states. Tensile behaviors of the damaged concrete specimen, including weight loss, stressstrain relationship, axial tensile strength and elastic modulus, are carried out. Occurrence and evolution of the internal cracks, micro-structural developments of the damaged concrete specimens were obtained as well. Based on the CT images of the specimen slices, the concrete specimen is reconstructed by matlab software. Tensile behavior evolutions of the damaged concrete are well uncovered using multi-scale analysis.

\section{Experimental program}

\subsection{Materials}

In this survey, commercial concrete is used to reduce the discreteness caused in the production process. The details of concrete mixtures are shown in Table 1. The cement type is P.O $42.5 R$. Dumbbell-shaped specimens were fabricated for the tension test, as shown in Fig. 1.

\subsection{Techniques and procedures}

\subsubsection{Experiment methods}

In-servicing, structures must be subjected to a variety of exposure conditions, such as chemical attack, drying and wetting cycles, temperatures, superficial carbonation, drying shrinkage and so on. All the conditions will affect the deteriorating process of concrete. Hence, it is difficult to exactly duplicate the field attack condition in the laboratory. Although the field test can better simulate the actual damage process, it always took much time to arrive at the deteriorating process (Kanazu 2001). Most of the present investigators select accelerated corrosion tests. Immersing and spraying 


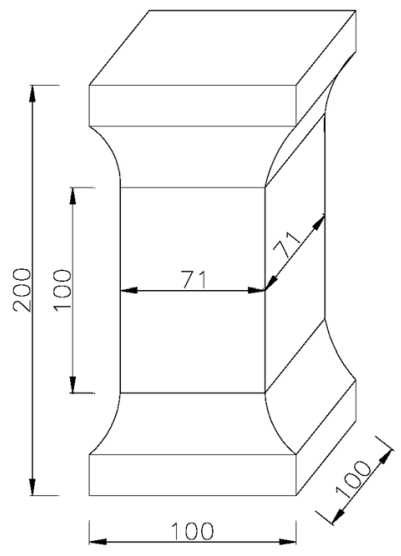

Fig. 1 Dimension of tensile concrete specimen (unit: $\mathrm{mm}$ )

Table 1 Mix proportion of the concrete mixtures

\begin{tabular}{ccccc}
\hline \hline Cement $\left(\mathrm{kg} / \mathrm{m}^{3}\right)$ & Sand $\left(\mathrm{kg} / \mathrm{m}^{3}\right)$ & Coarse aggregate $\left(\mathrm{kg} / \mathrm{m}^{3}\right)$ & Water $\left(\mathrm{kg} / \mathrm{m}^{3}\right)$ & Ratio $(\mathrm{w} / \mathrm{c})$ \\
\hline 283 & 784 & 1039 & 187 & 0.43 \\
\hline
\end{tabular}

methods are the two main ways to simulating the damage to construction materials done by acid rain in the laboratory. The spraying method is much closer to the natural process, while the immersing method is much faster. Through the comparison test, it is illustrated that both the methods are comparable and the results are reliable (Xie 2004). Accordingly, the immersing method is a reasonable choice for the accelerated test in this paper.

Generally, acid rain was caused by the acidic pollutants of $\mathrm{SO}_{2}$ and $\mathrm{NO}_{\mathrm{x}}$, and the $\mathrm{pH}$ level of acid rain is below 5.6. Considering that in large parts of China, the acid rain is due to sulfuric acid (Wang 2002), sulfuric acid type acid rain is simulated herein. Acid solution with $\mathrm{pH} 1.0$ was deposed by the mixture of sulfate and nitric acid. The $\mathrm{pH}$ value of the acid solution was detected by a digital $\mathrm{pH}$ meter (Fan 2010). To keep the $\mathrm{pH}$ level of the mixed solution constant, nitric acid solution were added periodically. At the same time, the solution was stirred thoroughly to reduce differential concentrations of the acid inside the solution tank.

\subsubsection{Specimen preparation}

A corrosion-resisting rectangular tank was used to contain acid solution and the concrete specimens. After curing for 28 days, the specimens were divided into two groups. One batch of specimens was continuously cured in water with $\mathrm{pH}$ value of 7.0, which was taken as a controlled group and was denoted as series $T 1$. The other batch of specimens was immersed in the anticorrosive tank filled with $\mathrm{pH} 1.0$ acid solution completely, and was denoted as series $T 2$. Exposure protocols were listed in detail in Table 2.

\subsubsection{Testing device}

After grinding the four side surfaces of the specimen with sand paper, strain gauges were attached onto each surface of the specimen on both vertical and transversal direction by JE-101 AB adhesive. 
Table 2 Conditioning details

\begin{tabular}{|c|c|c|c|c|}
\hline Designation & $\begin{array}{l}\text { Exposing } \\
\text { condition }\end{array}$ & $\begin{array}{l}\text { Solution } \\
\text { acidity }\end{array}$ & Specimen grouping & Immersion time (days) \\
\hline Series $T 1$ & pure water & $\mathrm{pH} 7.0$ & 3 specimens in a group, 8 groups & $0,10,20,30,40,50,60,70$ \\
\hline Series $T 2$ & $\begin{array}{c}\mathrm{H}_{2} \mathrm{SO}_{4}+\mathrm{HNO}_{3} \\
\text { (molar ratio is } 9: 1 \text { ) }\end{array}$ & pH 1.0 & 3 specimens in a group, 8 groups & $0,10,20,30,40,50,60,70$ \\
\hline
\end{tabular}

The dimension of the strain gauge is $50 \mathrm{~mm} \times 5 \mathrm{~mm}$, and the sensitivity factor of the strain gauge is $2.05 \pm 0.23 \%$. To obtain the displacement of the specimen, YHC-50 displacement sensors were placed on the side surface of the specimen as well.

MTS810 electro hydraulic servo universal testing machine was used for the tensile test of the concrete specimen. Both ends of the specimen were adhered to the steel plates by JGN-II adhesive that is widely used in building construction in China. Tensile and shear strength of JGN-II adhesive is greater than $35 \mathrm{MPa}$ and $18 \mathrm{MPa}$ respectively. Two steel plates were fixed to the upper and lower heads of the testing machine with 8 bolts $(18 \mathrm{~mm}$ in diameter). In the experiment, the static strain loading rate was $10^{-5} / \mathrm{s}$.

\subsubsection{Testing procedure}

After being immersed in the acid solutions at the scheduled time listed in Table 2, three specimens as one batch were taken out. The removed specimens were first dried for about two to three days, followed by the physical and tensile properties test (including weighting, CT, tensile test and SEM/EDS analysis). Macro mechanical properties (e.g. mass, tensile strength, tensile stressstrain relation and elastic modulus) of the concrete specimens under different damage states were examined respectively. Microstructures of the corroded specimens were discussed as well.

\section{Results and discussions}

3.1 Macro-mechanical property of the concrete specimen immersed in simulated acid rain environment

\subsubsection{Surface description}

During the test, specimens were periodically taken out from the acid solutions for visual inspection. It was observed that as conditioning continued, surface color gradually changed from gray to gray-black, then yellow and finally yellow-brown. Honeycomb voids were formed, mortar surface were spalling and coarse aggregates were exposed. It was discovered that the damage started from the specimen corners, and that the specimens became loose and gradually powdery (shown in Fig. 2).

\subsubsection{Mechanical characteristics}

(1) Weight loss

Based on the visual observations described above, it was obvious that honeycomb voids were formed as conditioning continued, causing the mass change. This could be measured by an electronic scale with an accuracy of $0.1 \mathrm{~g}$. The mass change of concrete specimen is defined as 


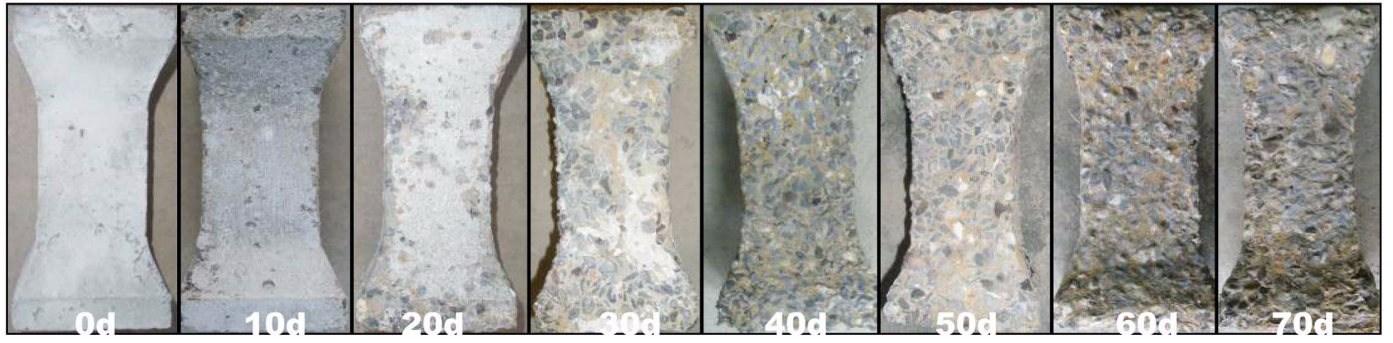

Fig. 2 Surface appearance of concrete specimen exposed to acid solution for various periods

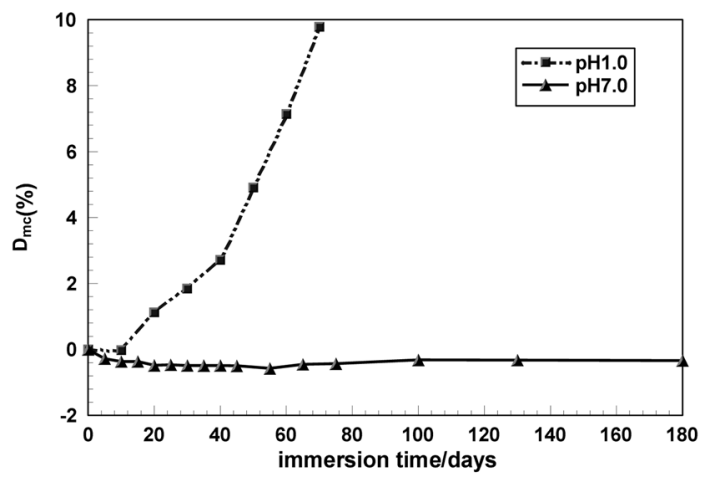

Fig. 3 Relation between mass loss ratio and immersion time

$$
D_{m t}=\left(1-m_{t t} / m_{t 0}\right) \times 100 \%
$$

Where, $D_{m t}$ is weight loss ratio of concrete specimens conditioned in acid solution, $m_{t t}$ is the mass of concrete specimens immersed in the acid solution for $t$ days; $m_{t 0}$ is the mass of concrete specimens cured in water with $\mathrm{pH}$ 7.0. The relations between weight losses ratio $D_{m t}$ and immersion time $t$ for the concrete specimen conditioned in acid solution and water are obtained respectively, which is shown in Fig. 3.

From Fig. 3, it is clear that the specimens show a steady rate of weight loss when exposed to the acid solutions. The rate of weight loss goes up gradually as the immersion time increases. However, the mass of specimen cured in water will somewhat increase due to the hydration process.

\section{(2) Tensile stress-strain curve}

It is well known that concrete is a kind of brittle material. Once the tensile cracking occurs, the specimen will fracture in a sudden way. Therefore, it is very difficult to record the complete stressstrain curve, and the softening phase is always lost. Stress-strain relations of the concrete specimen under different damage states are carried out, which is shown in Fig. 4(a). The relation between the ultimate strains at the peak stress as a function of immersion time can be derived as well (shown in Fig. 4(b).

For the concrete specimens under different damage states, the shapes of the stress-strain relation curves are close to each other. Moreover, as the damage continues, the tensile strength and elastic modulus will decrease gradually. The ultimate strain at the peak stress increase at the initial 

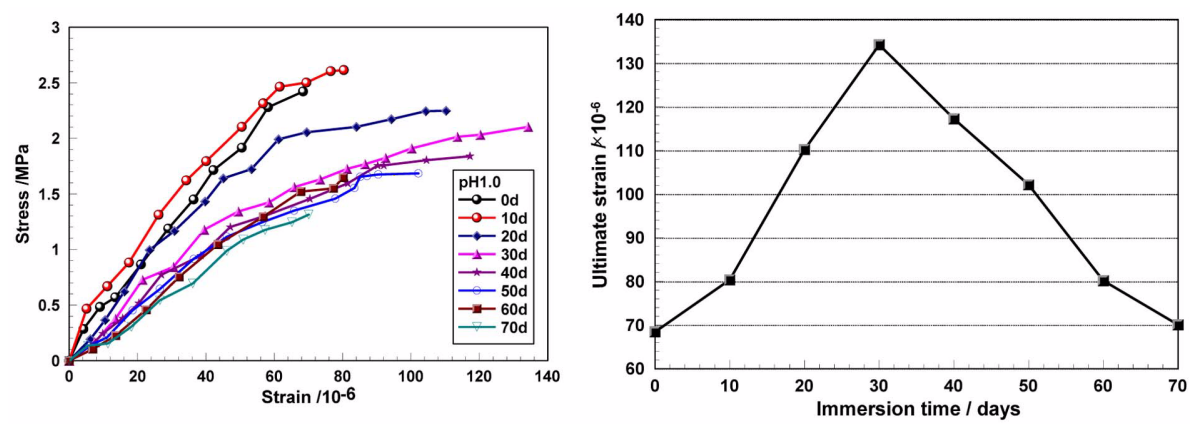

Fig. 4 Stress-strain curve of concrete specimen immersed in simulated acid rain environment for various periods: (a) Stress-strain relation, (b) strain at the peak stress

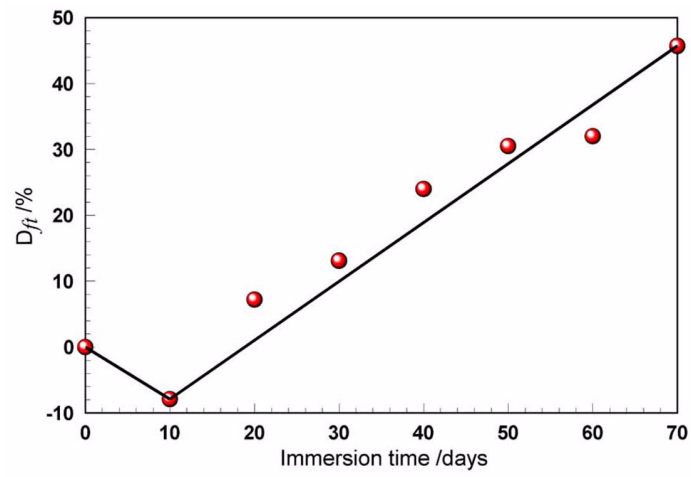

Fig. 5 Relation between immersion time and tensile strength of damaged concrete

immersion periods (less than 30 days) and will decrease later on. That is, the concrete will become a little more ductile at the initial damage period. However, once the damage becomes more severe, it will become more and more brittle distinctly.

(3) Tensile strength

To compare the tensile strength deteriorating trend of the concrete specimen submerged in acid solutions, relative tensile strength variation ratio is defined as

$$
D_{f t}=\left(1-f_{t t} / f_{t 0}\right) \times 100 \%
$$

Where, $D_{f t}$ is the relative strength degradation ratio of axial tensile strength for deteriorated concrete specimen, $f_{t t}$ are the tensile strength of concrete exposed to the acid solution for $t$ days; and $f_{t 0}$ is the tensile strength of the controlled specimens cured in the water at the same age. Relation between $D_{f t}$ and the immersion time is plotted in Fig. 5.

Fig. 5 reports mean values for tensile strength of three concrete specimens tested at various immersion times. The figure shows that the controlled specimens conditioned in water are gaining strength with the increase of the immersion time. However, the trend is different from those conditioned in the acid solutions. The tensile strength of the concrete samples takes a slight increase 


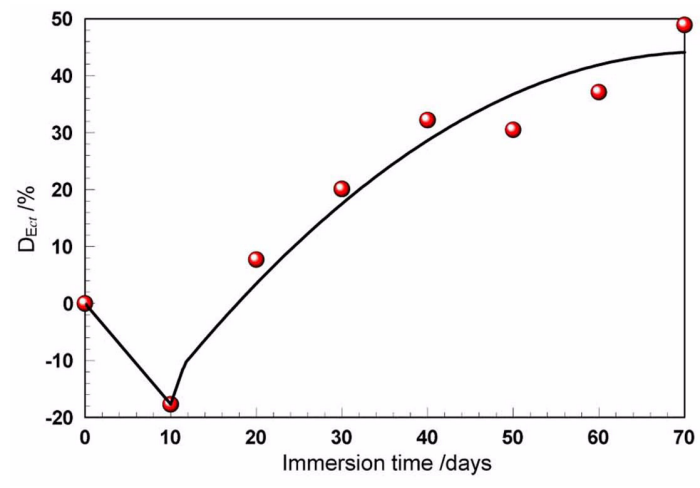

Fig. 6 Relation between immersion time and tensile elastic modulus of corroded concrete

at the initial damage stage, while it will decrease once the immersion time reaches a critical value. Relation between $D_{f t}$ and immersion time is linear. The critical time is determined by the acidity of the solution, which will decrease with the increase of acidity.

\section{(4) Tensile elastic modulus}

During the tensile process, the relation between the strain and stress is in the elastic regime until the stress reach 40 to 60 percent of the ultimate tensile stress. Based on reported research, secantmodule corresponding of the peak stress is considered as the elastic modulus of concrete. To well describe the deteriorating trend of tensile elastic modulus of the concrete specimen submerged in acid solutions, the relative elastic modulus variation ratio is defined as

$$
D_{E t}=\left(1-E_{t t} / E_{t 0}\right) \times 100 \%
$$

Where, $D_{E t}$ represent the relative elastic modulus degradation ratio for damaged concrete specimen, $E_{t t}$ is the tensile elastic modulus of concrete specimen corroded for $t$ days, $E_{t 0}$ is the tensile elastic modulus of the controlled specimen. Relation between the tensile elastic modulus of corroded concrete specimen and the immersion time is shown in Fig. 6.

From Fig. 6, it is shown that in the initial damage stage, the elastic modulus of concrete exhibits a slight increase. When the damage continues, the elastic modulus of the specimen gradually decreases. After being immersed in the acid solution for 70 days, the elastic modulus will decrease about 49 percent of the controlled specimen.

\section{(5) Failure process}

Failure processes under tensile test for the specimens which have deteriorated using different acid solutions are recorded in the experimental process. For the 3 concrete specimens in each testing group (listed in Table 2), the failure modes and the fracture surfaces are identical to each other completely. The failure modes, and the fracture surfaces in the failure state, for the concrete specimens exposed to acid solutions for various periods, are shown in Fig. 7.

From Fig. 7, it can be seen that all the specimens failed in the middle part of the specimen, tensile fractures are ensured in this study. It is also discovered that the specimen becomes more brittle if the damage becomes severe. It is clear that the fracture surfaces are different from each other for 
(a)

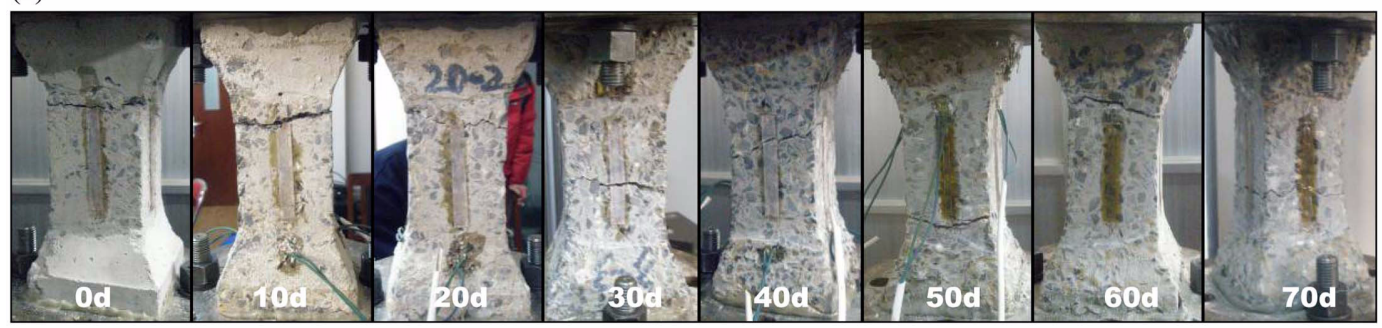

(b)

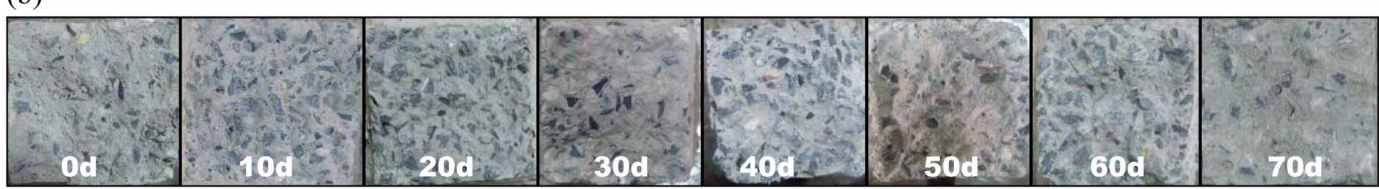

Fig. 7 Typical fracture pattern of concrete under different damage states: (a) Failure mode, (b) Fracture surface

the specimens under different damage states. The failure modes change from fracture of cement or aggregate material, to the interfacial fracture between mortar and aggregate. Therefore, it can be derived that change of failure mode is caused by the degradation of the bond property between mortar and aggregate. The bond strength between mortar and aggregate can be measure by the technology of Nano-indentation.

\subsection{Observation of changes in microstructure of concrete by CT images}

The microstructure of concrete greatly influences its permeability, strength and durability. The mechanical characteristics of the concrete are greatly dependent on its internal structure. Here, to well understand the damage mechanism of concrete, it is essential to discover its microstructure under different damage states.

Since the X-rays are absorbed by the concrete specimen according to composition and density of the material, different features can be detected. Objects with a higher density absorb more X-rays, resulting in bright areas. Consequently, the lower the density, the less X-rays is absorbed, creating darker regions. Hence, CT was used to determine the meso-level structure of concrete specimen under different damage states herein.

\subsubsection{Test procedure}

After curing the concrete prism specimen in water for 28 days, it was taken out and scanned. Each scan was $1 \mathrm{~mm}$ thick, and the total number of the scans was about 200. The internal mesolevel structure of the specimen is specified based on the 200 scanning images. Then, the specimens were conditioned in the simulated acid rain for ccarious periods and scanned again.

For the measurements, a 'Siemens Somatom Sensation' 16-slice spiral computed tomography scanner made in Germany was used. Samples were scanned with a fixed X-ray source, at $140 \mathrm{kV}$, $200 \mathrm{~mA}$ and $22.60 \mathrm{mGy}$ CTD. Each scan was $1 \mathrm{~mm}$ thick and the total number of the scans was about 200 . 


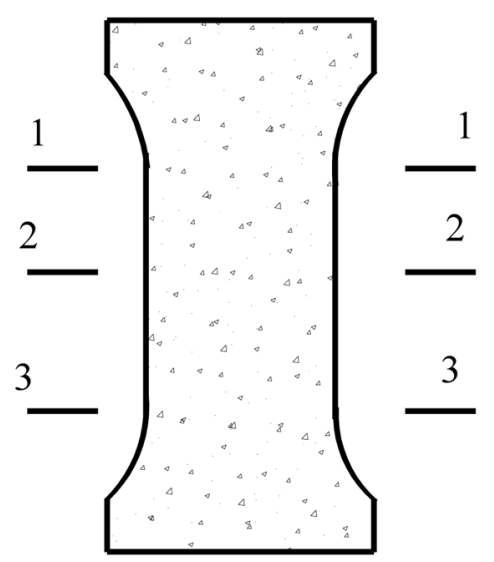

Fig. 8 Location of scanning on concrete specimen

\subsubsection{CT images}

From CT test, 200 scanning images were obtained for every concrete specimen. Scanning images at the three cross-sections (plotted in Fig. 8) of concrete exposed to the simulated acid solution for 0,10 and 20 days are given in this paper (shown in Fig. 9).

From Fig. 9, it can be seen that damage originated from the surface. With the immersion continues, more and more concrete on the lateral sides of the specimen are gradually spalled. During the whole deteriorating process, the development of micro cracks in the samples can be observed. It is also shown that the voids in the specimens become wider and bigger with the corrosion continues.

\subsubsection{Explain the weight loss of damaged concrete by CT images}

From the CT test, it is clear that when the damage continues, the CT images become darker and darker. Based on the theory of CT technology, it can be concluded that with continuing damage, the density of concrete specimen becomes lower. This result is in good agreement with the weighing test, which is shown in Fig. 4.

\subsubsection{D reconstruction of the concrete specimen based on the scanning images}

Since the behavior of concrete is exceedingly complex, numerical simulations coupled with theory and experiment, are considered to be an extremely important tool for examining material properties successfully (Wriggers 2006). Whether the generated numerical model can actually reflect the real structure is decided by the results of the numerical computation. Some algorithms for generating realistic concrete models are proposed based on stochastic theory. From the images shown in Fig. 10 , the location, shape and size of the voids and aggregates can be exactly determined. Based on the 200 scanning images obtained in the CT test, 3D structure of the specimen is reconstructed by a program of MATLAB (shown in Fig. 10).

From Fig. 10, it can be seen that the specimen surface become coarser when the damage continues, which agrees with the visual observation.

Therefore, CT is an efficient technology to explain the macro mechanical properties of concrete specimen (such as surface appearance, weight loss) qualitatively from the meso-scale. Simultaneously, it can provide a most direct and convenient way to build up the digital model for 


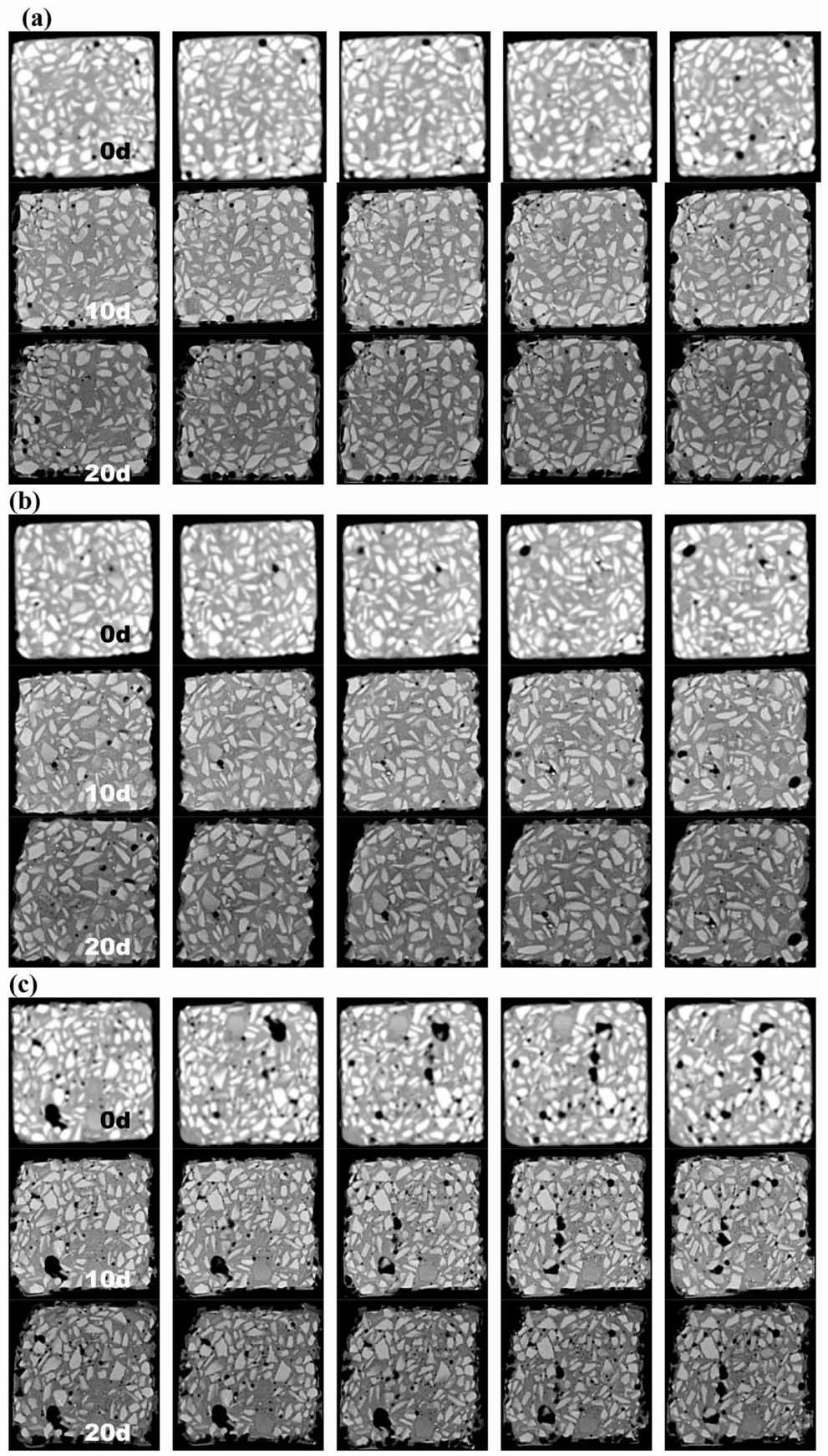

Fig. 9 Comparison of CT images of specimen exposed to acid solution for various periods: (a) Scanning layer of cross-section 1-1; (b) Scanning layer of cross-section 2-2, (c) Scanning layer of cross-section of 3-3

the numerical computation used to describe the mechanical property of deteriorated concrete specimen. The reconstructed structure of the concrete can reflect the real structure well, and is better than the models generated by stochastic methods. 


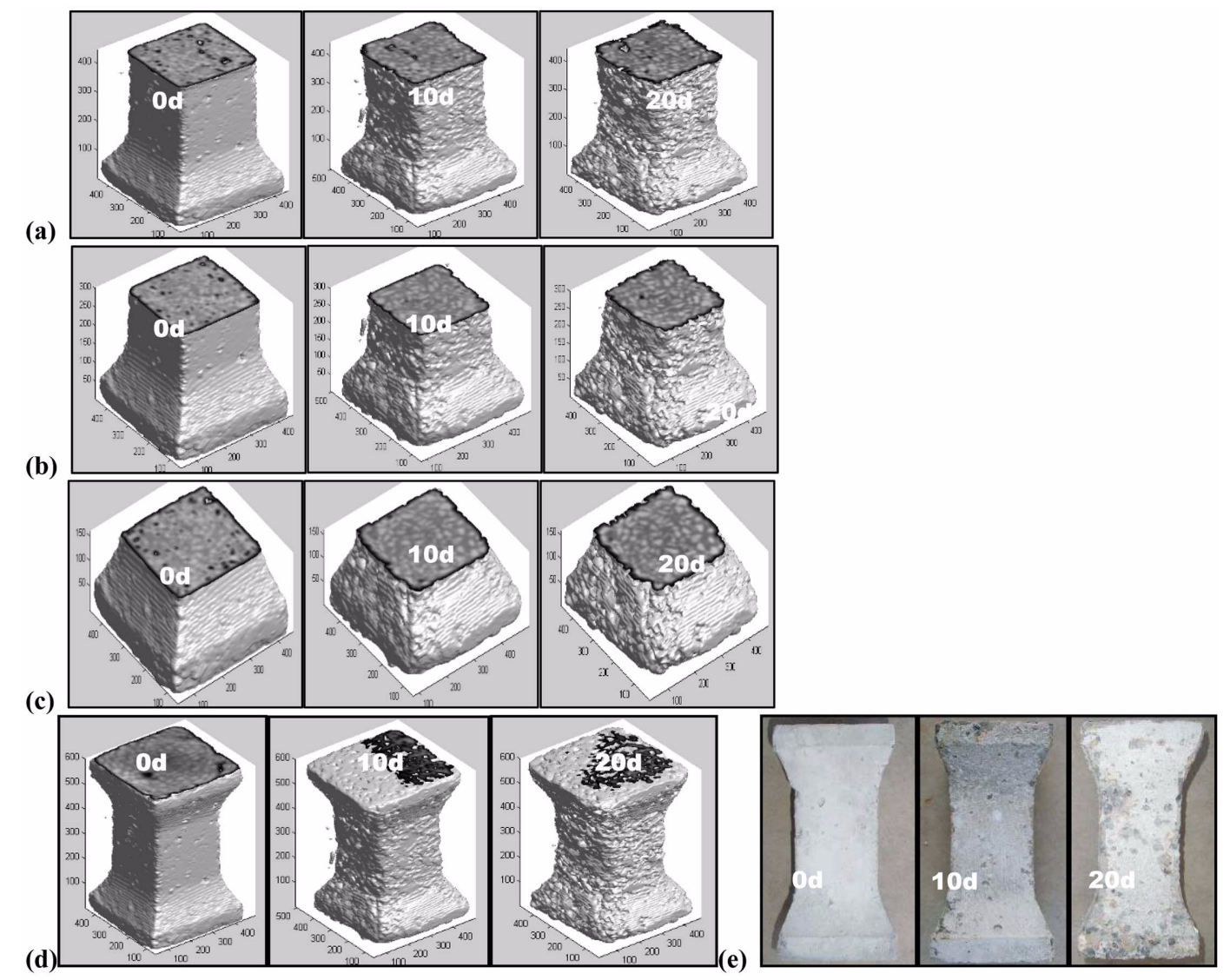

Fig. 10 3D reconstruction of concrete specimen: (a) Reconstruction of concrete specimen_section 1-1, (b) Reconstruction of concrete specimen_section 2-2, (c) Reconstruction of concrete specimen_section 3-3, (d) Reconstruction of concrete specimen under different damage states, (e) Surface of the concrete specimen under different damage states

\subsection{SEM/EDS analysis of microstructure of concrete}

To reach a good understanding of the development of voids and internal micro cracks, and change of composition in the deteriorated concrete specimen, SEM and EDS analyses were performed on the concrete samples at different damage stages.

\subsubsection{Sample preparation}

Samples of $1 \mathrm{~cm}^{3}$ are prepared for the SEM test. Three samples are cut from the concrete specimen. Since the specimen is small, acidic solution with $\mathrm{pH} 2.5$ was deposed by the mixture of sulfate and nitric acid by acid solution herein. Micro-structures of the samples immersed in the acidic solution for 0 day, 3 days, 7 days and 10 days are analyzed by JSM-6360LV SEM. Evolutions of the voids, micro morphology, chemical composition, cracking in the samples with immersion time are examined respectively. 
Table 3 Porosity property of the concrete

\begin{tabular}{|c|c|c|c|c|c|c|c|c|}
\hline No. & $\begin{array}{l}\text { Total mercury } \\
\text { volume } / \mathrm{ml} / \mathrm{g}\end{array}$ & $\begin{array}{c}\text { Total } \\
\text { specific } \\
\text { surface area } \\
\text { in hole } / \mathrm{m}^{2} / \mathrm{g}\end{array}$ & $\begin{array}{l}\text { Medium hole } \\
\text { diameter } \\
\text { volume/nm }\end{array}$ & $\begin{array}{l}\text { Medium hole } \\
\text { diameter } \\
\text { areas/nm }\end{array}$ & $\begin{array}{c}\text { Skeleton } \\
\text { density } \\
\text { /g/ml }\end{array}$ & $\begin{array}{c}\text { Apparent } \\
\text { density at } \\
0.52 \text { psia } \\
/ \mathrm{g} / \mathrm{ml}\end{array}$ & $\begin{array}{c}\text { Porosity } \\
\text { ratio/\% } \%\end{array}$ & $\begin{array}{c}\text { Average } \\
\text { porediameter } \\
\text { /nm }\end{array}$ \\
\hline 1 & 0.0771 & 6.729 & 90.4 & 19.1 & 2.4198 & 2.0393 & 15.7247 & 45.8 \\
\hline 2 & 0.1016 & 7.841 & 159.5 & 17.9 & 2.3874 & 1.9214 & 19.5213 & 51.8 \\
\hline 3 & 0.0949 & 8.582 & 128.5 & 15.3 & 2.4139 & 1.9639 & 18.6412 & 44.2 \\
\hline
\end{tabular}

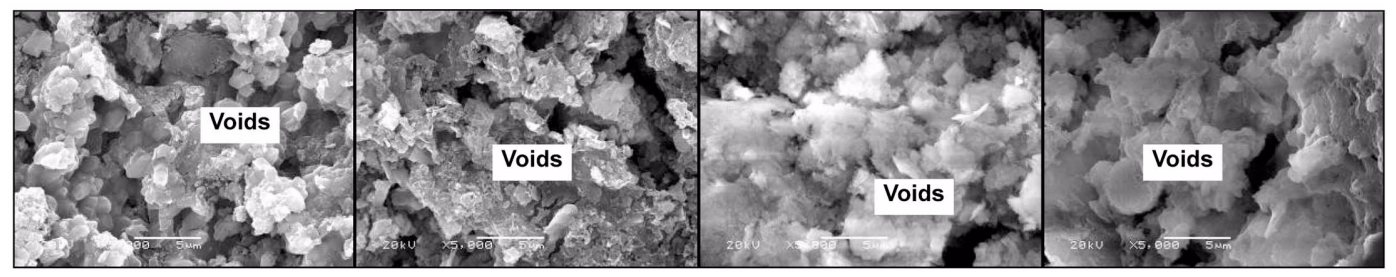

Fig. 11 SEM image of voids formed in concrete specimen

\subsubsection{Mercury intrusion porosimetry (MIP)}

The pore volume and structure in the material affect its permeability, which will influence its durability. To determine the pore characteristics in the testing concrete samples, mercury intrusion tests were executed on three samples in advance. Table 3 presents the porosity characteristics for the testing samples.

\subsubsection{Evolution of voids}

Voids can be observed by SEM technology. The voids characteristics in the concrete samples under different damage states are shown in Fig. 11.

From the SEM images, it can be seen that the voids become bigger and wider. Once the concrete specimen was damaged in acid medium, the volume of voids will increase. Based on the theory of meso-mechanics, concrete can be considered as a combined material which is consisted of base material and void. Relation between the volume of base material and voids can be expressed as

$$
C_{1}=\left(m_{t 0}-m_{t t}\right) / m_{t t}, \quad C_{1}+C_{0}=1
$$

Where, $C_{1}$ is the volume of voids and $\mathrm{C}_{0}$ is the volume of base material.

In this way, the relation between the developments of voids volume over time can be obtained quantitatively, which is shown in Fig. 12.

\subsubsection{Evolution of micro cracks}

Evolutions of micro cracks in the specimen were detected by SEM. The micro cracks in the samples under different damage states are shown in Figs. 13-14.

For the controlled samples, only short, thin micro cracks can be seen, few transverse cracks are found. Whereas, for the samples being immersed in the acidic solution till three days, internal micro cracks occurred, and the internal micro cracks become gradually wider. With the damage continues, the internal cracks become wider, and the new micro cracks are formed by the adjacent voids. 


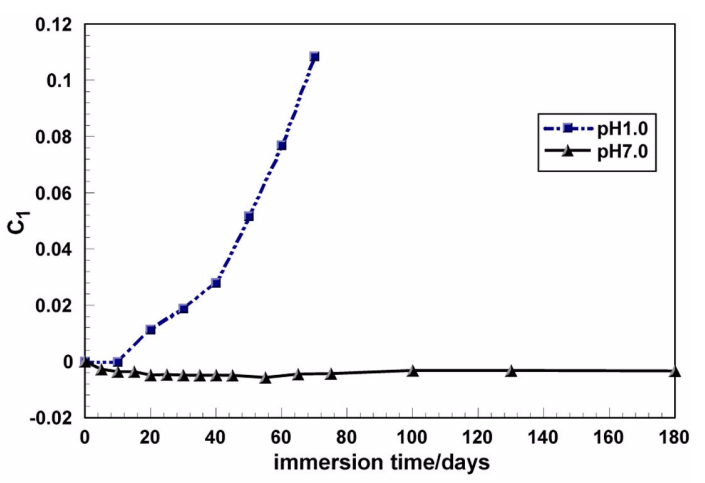

Fig. 12 Relation between void volume and immersion time

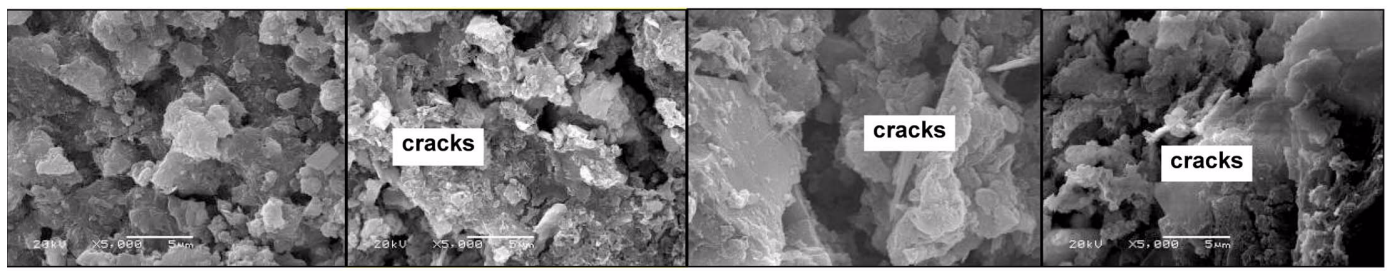

Fig. 13 SEM image of cracking formed in concrete specimen

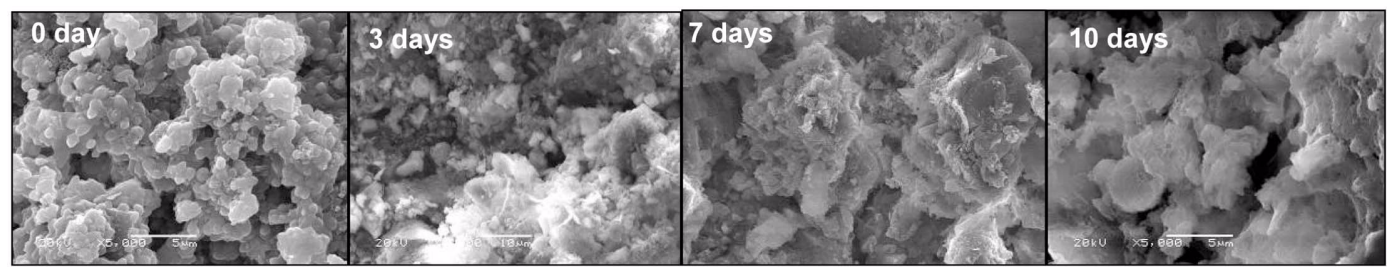

Fig. 14 SEM micrographs of concrete under different damage states

\subsubsection{Morphology analysis}

The damage to concrete in acidic solution is mainly caused by the complex chemical and physical reactions. When the damage continues, the chemical compositions in the samples change. Microstructures of concrete samples exposed to the acid solution for 0 day, 3 days and 7 days are shown in Fig. 15 respectively.

From the morphology analysis of the internal structure of concrete sample, it is clear that the chemical compounds have changed. With the increase of the immersion time, the chemical reactions occur between the cement and the acidic medium, new chemical compounds are generated gradually. Accordingly, the damage mechanism of the concrete in acidic solution can be well understood by SEM analysis.

\subsubsection{EDS analysis: Distribution of chemical elements in the samples}

EDS is one of the variants of X-ray fluorescence spectroscopy which relies on the investigation of a specimen through interactions between electromagnetic radiation and matter. The spectroscopic 
(a)
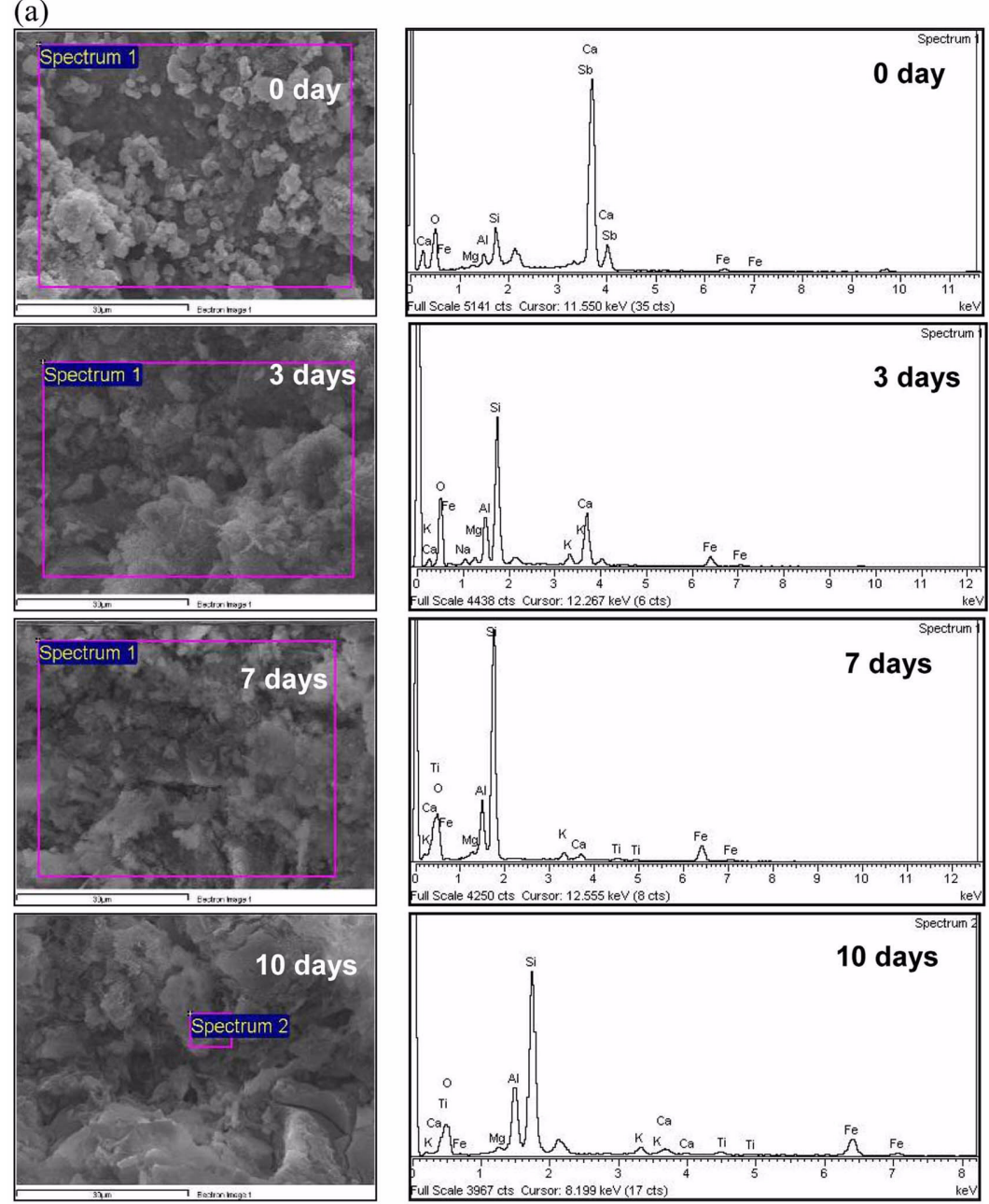

(b)

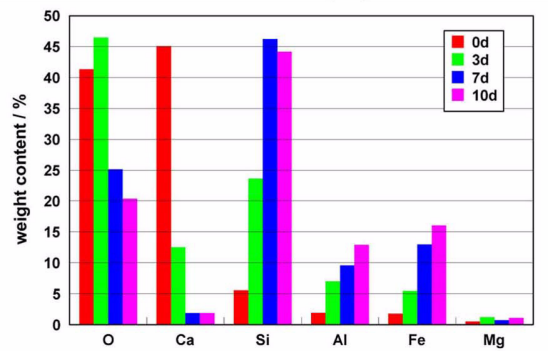

Fig. 15 SEM/EDS spectrum of concrete specimen under different damage states: (a) EDS spectra of concrete specimen under different damage states, (b) Evolution of weight contents of chemical elements

technique allowed the mapping of the elemental distribution within the samples. In this connection in different damage states, the analytical technique was used to carry out the elemental analysis and the chemical characterization of the concrete samples. The EDS spectra and elemental contents are shown in Fig. 15(b). 
As determined by EDS, the predominant elements in the concrete samples were $\mathrm{O}, \mathrm{Ca}, \mathrm{Si}$ and $\mathrm{Al}$ in various compounds. When the damage caused by acidic solution continues, the content of $\mathrm{Ca}$ shows significant reduction, whereas the content of $\mathrm{Si}$ and $\mathrm{Al}$ shows an obvious increase. This result is in good agreement with the interpretation of deteriorating mechanism of concrete in acid medium reported by other researches (Brown 1988, Marchand 1999, Metha 2006). That is, more soluble products are formed through the chemical reaction between and nitric acid, sulfate acid and calcium hydroxide. Therefore, calcium compounds of cement paste formed in concrete through the hydration process, and the calcium in calcareous aggregate, are both leached away, and silica gel or sometimes magnesium silicate hydrate fill the space. This is the fundamental reason that leads to the degradation of macro mechanical behaviors of concrete.

\section{Conclusions}

To discover the tensile property of concrete attacked by acid rain solution, comprehensive experiments were conducted on dumbbell-shaped concrete specimens from different space scales respectively. Pure water and acid solutions mixed by sulfate and nitric acid solutions with $\mathrm{pH}$ level of 1.0, are selected for accelerated conditioning. A series of tests, including physical and tensile property tests, MIP, CT, SEM/EDS were performed on the concrete specimen in different prepared states. Weight loss, voids property, tensile stress-strain relation, axial tensile strength, meso and micro structure in the concrete specimens under different damage states are examined quantitatively. The results obtained on different scales are compared and discussed in detail. The macro phenomena and deteriorating mechanism of the concrete specimen polluted by acidic solution are well explained by the results obtained on the macro, meso and micro scale. The following conclusions can be drawn:

(1) The specimens show a steady rate of weight loss when exposed to the acid solutions. The rate of weight loss gradually goes up with the increase of immersion time. Based on the theory of meso-composite mechanics, the relation between voids volume with time is built up.

(2) Three damage indices, $\mathrm{D}_{m t}, \mathrm{D}_{f t}$ and $\mathrm{D}_{E t}$, corresponding to relative weight loss ratio, tensile strength variation and elastic modulus variation are defined and calculated using the test results. It is concluded that all the three indices decrease in the initial immersion period (less than ten days), and increase obviously when the immersion continues.

(3) It was shown that CT technology is an efficient way to discover the evolution of the internal structure of concrete on a meso scale. Based on the images, the numerical model is reconstructed. It is evident that the reconstructed numerical specimen gives a more accurate description of internal structure in concrete than the random aggregate model.

(4) It is shown that the ultimate strain at the peak stress increase slightly at the initial immersion periods (less than 30 days), and decrease later on. Therefore, the ductility of concrete will have a slight increase at the initial damage period. However, once the damage continues, the concrete will become more brittle.

(5) From SEM/EDS tests, it is concluded that the content of the element Ca exhibits a significant reduction when the immersion continues, whereas the content of element $\mathrm{Si}$ and $\mathrm{Al}$ obviously increases. More soluble products are formed and have been leached away when the damage continues. This is the fundamental reason that leads to the degradation of macro mechanical behaviors of concrete. 


\section{Acknowledgements}

This work was funded by the National Science Foundation of P.R. China (Grant No. 51178069), National Science Foundation of P.R. China (Grant No. 50708010) and Liaoning Provincial Funded project (Grant No. 20092149). We thank B. Movaghar, the research professor of Northwestern University, USA, for reviewing this paper.

\section{References}

Brown, P.W. and Clifton, J.R. (1988), "Mechanism of deterioration in cement-based materials and lime mortar", Durability Build. Mater., 5(3-4), 409-420.

Cnudde, V., Cwirzen, A., Masschaele, B. and Jacobs, P.J.S. (2009), "Porosity and microstructure characterization of building stones and concretes", Eng. Geol., 103(3-4), 76-83.

Fan, Y.F., Hu, Z.Q., Zhang, Y.Z. and Liu, J.L. (2010), "Deterioration of compressive property of concrete under simulated acid rain environment", Constr. Build. Mater., 24(10), 1975-1983.

Fattuhi, N.I. and Hughes B.P. (1998), "The performance of cement paste and concrete subjected to sulphuric acid attack", Cement Concrete Res., 18(4), 545-553.

Girardi, F., Vaona, W. and Maggio, R.D. (2010), "Resistance of different types of concretes to cyclic sulfuric acid and sodium sulfate attack", Cement Concrete Comp., 32(8), 595-602.

Grattan-Bellew, P.E. (1996), "Microstructural investigation of deteriorated Portland cement concretes", Constr. Build. Mater., 10(1), 3-16.

Huang, P., Bao, Y. and Yao, Y. (2005), "Influence of $\mathrm{HCl}$ corrosion on the mechanical properties of concrete", Cement Concrete Res., 35(3), 584-589.

Ito, M., Mitchell, M.J. and Driscoll, C.T. (2000), "Spatial patterns of precipitation quantity and chemistry and air temperature in the Adirondack region of New York", Atmos. Environ., 36(6), 1051-1062.

Kanazu, T., Matsumura, T., Nishiuchi, T. and Yamamoto, T. (2001), "Effect of simulated acid rain on deterioration of concrete", Water Air Soil Poll., 130(1-4), 1481-1486.

Kong, H. and James, G. (1987), "Concrete deterioration due to acid precipitation", ACI Mater. J., 84(2), 110-116.

Marchand, J. and Skalny, J.P. (1999), Materials science of concrete: sulfate attack mechanism, Ed. American Ceramic Society, USA.

Menz, F. and Seip, H. (2004), "Acid rain in Europe and the United States: an update”, Environ. Sci. Policy, 7(4), 253-265.

Metha, P.K. and Monteiro, P.J.M. (2006), Concrete: microstructure, properties, and materials, Ed. McGraw-Hill, New York.

Pavlik, V. (1994), "Corrosion of hardened cement paste by acetic and nitric acids Part I: calculation of corrosion depth", Cement Concrete Res., 24(3), 551-562.

Rendell, F. and Jauberthie, R. (1999), "The deterioration of mortar in sulphate environments", Constr. Build. Mater., 13(6), 321-327.

Sersale, R., Frigione, G. and Onavita, L. (1998), "Acid depositions and concrete attack: main influences", Cement Concrete Res., 28(1), 19-24.

Wang, T.J., Jin, L.S., Li, Z.K. and Lam, K.S. (2002), "A modeling study on acid rain and recommended emission control strategies in China", Atmos. Environ., 34(26), 4467-4477.

Wriggers, P. and Moftah, S.O. (2006), "Mesoscale models for concrete: homogenisation and damage behavior", Finite Elem. Anal. Des., 42(7), 623-636.

Xie, S., Qi, L. and Zhou, D. (2004), "Investigation of the effects of acid rain on the deterioration of cement concrete using accelerated tests established in laboratory", Atmos. Environ., 38(27), 4457-4466.

Zhang, Y.L., Lee, X.Q. and Cao, F. (2011), "Chemical characteristics and sources of organic acids in precipitation at a semi-urban site in Southwest China", Atmos. Environ., 45(2), 413-419.

Zivica, V. and Bajza, A. (2002), "Acidic attack of cement-based materials-a review Part 2. Factors of rate of acidic attack and protective measures", Constr. Build. Mater., 16(4), 215-222.

Zivica, V. and Bajza, A. (2004), "Acidic attack of cement based materials-a review Part 3: research and test methods", Constr. Build. Mater., 18(9), 683-688. 